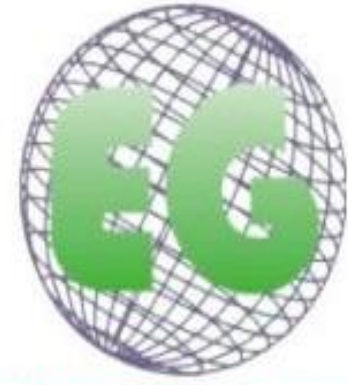

ISSN 1695-6141 $N^{\circ} 46$

\title{
Análisis de impacto de la crisis económica sobre el síndrome de Burnout y resiliencia en el personal de enfermería
}

Analysis of impact of the economic crisis on the syndrome of Burnout and resilience in nursing

\section{*Muñoz Sánchez, Javier **Martínez, Noelia del Amor ***Lázaro Sahuquillo, Marta ${ }^{* * * *}$ Carranza Román, Aurora ${ }^{* * * * *}$ Martinez Cantó, Marta}

*Enfermero. Unidad de Salud Mental Infanto Juvenil Area de salud de Ibiza y Formentera. Ibiza.

**Enfermera. Hospital Can Misses. Área de salud de Ibiza y Formentera. Ibiza. E-maill: noeliadam@hotmail.com ***Enfermera. Hospital La Mancha Centro, Alcazar de San Juan. Ciudad Real. ****Enfermera. Hospital Perpetuo Socorro. Albacete. ****Enfermera. Hospital Morales Meseguer, Murcia. España.

http://dx.doi.org/10.6018/eglobal.16.2.239681

\section{RESUMEN}

Este proyecto de investigación pretende analizar las condiciones de trabajo, las variables individuales y de salud que están relacionadas con el desarrollo del síndrome de burnout, así como el potencial efecto de la "entereza" o "personalidad resistente", presente en nuestro ámbito de trabajo, todo esto en el contexto de crisis económica en la que nos encontramos en la actualidad. Partiendo de este análisis, se podrían plantear estrategias de cambio con el fin de mejorar la satisfacción laboral y disminuir los niveles de desgaste laboral, actuando sobre los factores que más incidan sobre el mismo.

Se trata de un estudio observacional, descriptivo, transversal, realizado en una muestra de 377 profesionales de enfermería y auxiliares de enfermería, de Atención Especializada y Atención Sociosanitaria del Sistema Sanitario Público de la ciudad de Albacete. Los sujetos se seleccionarán de forma no aleatoria según disponibilidad en servicio/unidad de trabajo.

La prevalencia del síndrome de burnout es superior al periodo precrisis siendo el cansancio emocional la dimensión más influyente en su aparición. Existen ciertos factores que predisponen al aumento del burnout y otros que son factores de riesgo para su desarrollo. A su vez la personalidad resistente actúa como factor protector en la aparición del síndrome. Futuras líneas de investigación podrían estar encaminadas al estudio de intervenciones para el fomento de la resilencia.

Palabras clave: Crisis económica; Burnout; Resilencia; Enfermería. 


\section{ABSTRACT}

This research project aims to analyze the conditions of work, the individual variables and health are related to the development of burnout syndrome, as well as the potential effect of "hardness" or "resistant personality", present in our field of work, all this in the context of economic crisis in which we find ourselves today. Based on this analysis, strategies for change in order to improve job satisfaction and lower levels of work wear, acting on the factors that most influence on it could pose.

It is an observational, descriptive, transversal, study in a sample of 377 nursing professionals and auxiliary nurses specialized care and attention health public system socio-health of the city of Albacete. Subjects will be selected randomly according to availability in service/work unit.

The prevalence of burnout syndrome is superior to its pre-crisis being the emotional exhaustion the more influential in his appearance dimension. There are certain factors that predispose to the increase of burnout and others that are risk factors for its development. At the same time the resistant personality acts as a protective factor in the emergence of the syndrome. Future lines of research could be aimed at the study of interventions for the promotion of resilience.

Keywords: Economic crisis; Burnout; resilience; nursing.

\section{INTRODUCCIÓN}

La crisis financiera se caracteriza como un desorden, una perturbación, que afecta a variables financieras con tal intensidad que puede ponerse en peligro el funcionamiento razonablemente ordenado del sistema económico ${ }^{(1)}$. Una economía entra en recesión técnica cuando ha experimentado por dos trimestres consecutivos un crecimiento negativo del producto interior bruto (PIB) ${ }^{(2)}$.

Analizando el entorno español económico, podemos ver cómo la situación de crisis generalizada comenzó a manifestarse a finales de 2007. En el curso del año fiscal 2006 el PIB era del 4\%, en 2007 y 2008 descendió a 3,9\% y 2010 pasó a ser de 0,8\% (Fondo Monetario Internacional, Eurostat 2010) ${ }^{(3)}$.

Como consecuencia directa de esta situación financiera se producen recortes desproporcionados en los recursos y restricciones en la oferta de servicios, ello conduce a efectos potencialmente negativos sobre la igualdad, la eficiencia y la calidad de los servicios sanitarios ${ }^{(4)}$.

Tras décadas de trabajo de integración y de ampliación progresiva de la cobertura hasta alcanzar la universalidad de nuestro Sistema Nacional de Salud (SNS), se han producido modificaciones en la Ley General de Sanidad (1986), la financiación de la sanidad por impuestos (1999), las Leyes de Extranjería (2000), Cohesión (2003) y la Ley General de Salud Pública (2011) ${ }^{(5)}$.

El Real Decreto-Ley 16/2012, de 20 de abril, de medidas urgentes para garantizar la sostenibilidad del Sistema Nacional de Salud y mejorar la calidad y seguridad de sus prestaciones ${ }^{(4)}$, modifica elementos sustantivos de nuestro sistema sanitario como la Ley 16/2003, de 28 de mayo, de cohesión y calidad del Sistema Nacional de Salud; Ley 29/2006, de 26 de julio, de garantías y uso racional de los medicamentos y productos sanitarios; Real Decreto 823/2008, de 16 de mayo, por el que se establecen los márgenes, deducciones y descuentos correspondientes a la distribución y dispensación de medicamentos de uso humano; Ley 44/2003, de 21 de noviembre, de ordenación de las profesiones sanitarias; Ley 55/2003, de 16 de diciembre, del Estatuto Marco del personal estatutario de los servicios de salud; Ley Orgánica 4/2000, de 11 de enero, sobre derechos y libertades de los extranjeros en España y su integración social; Real Decreto 240/2007, de 16 de febrero, sobre 
entrada, libre circulación y residencia en España de ciudadanos de los Estados miembros de la Unión Europea y de otros Estados parte en el Acuerdo sobre el Espacio Económico Europeo ${ }^{(6)}$. El real decreto ley 20/2012, del 13 de julio, de medidas para garantizar la estabilidad presupuestaria y de fomento de la competitividad suprimió la paga extraordinaria del segundo semestre del año 2012 al personal estatutario y funcionario ${ }^{(7)}$.

El resto de las medidas contempladas por el decreto están relacionadas con la ordenación de la cartera de servicios, el establecimiento de fórmulas de copago para la prestación farmacéutica y prestaciones complementarias. Este decreto provoca una quiebra del modelo de universalidad ${ }^{(5)}$

Con el fin de obtener un panorama general de la situación actual en el SNS trataremos de identificar los principales elementos que pueden ser causantes de su actual crisis y sostenibilidad:

- El gasto sanitario en España es de los más bajos de la Unión Europea según la OMS ${ }^{(7,8)}$.

- El gasto farmacéutico en nuestro país es muy elevado (promedio del $20,4 \%$ sobre el $17,2 \%$ de la OECD).

- Gestión politizada y escasa atención a la cronicidad/dependencia y deficiente atención sociosanitaria.

- Deterioro de la Atención Primaria de Salud. Las actividades de promoción de la salud y prevención de la enfermedad han quedado relegadas (presupuesto en torno al $2 \%$ del gasto sanitario) al ser desplazadas por actividades asistenciales.

- Escaso desarrollo reglamentario de la Ley General de Sanidad. No se ha logrado la cohesión y coordinación deseables entre Comunidades Autónomas pese a la aprobación de la Ley de Cohesión Sanitaria ${ }^{(9) .}$

Las repercusiones de la crisis económica sobre la salud de la población general son un incremento de la mayoría de las enfermedades, con especial relevancia de las enfermedades y problemas de salud mental y un aumento de la mortalidad ligada al ascenso de las principales causas de muerte (enfermedades cardiovasculares, incremento del consumo de drogas, traumatismos y suicidios) ${ }^{(10)}$.

Esta situación económica ha repercutido sobre los profesionales de enfermería, de modo que, a partir de 2010, muchas administraciones aprovechan la situación para reducir los recursos humanos y materiales como medida inmediata de ahorro, generando sobrecarga laboral y empeoramiento de la atención que se presta en los centros sanitarios. Esta medida, sumada a otras impuestas como la reducción de salarios o congelación salarial, aumento de la jornada laboral, disminución de plantillas enfermeras, empeoramiento de la posibilidad, ya precaria, de conciliación de la vida laboral y personal, etc., están conformando un nuevo contexto para la enfermería española en el que se está actuando de manera directa sobre el entorno laboral, deteriorándolo y provocando desmotivación a nivel profesional ${ }^{(11)}$.

Si calculamos en 180.000 las enfermeras tituladas asistenciales en activo, la tasa de desempleo enfermero sería del $8 \%{ }^{(12)}$. Analizando los datos según los contratos también se ha observado un incremento de la precariedad laboral, pues los contratos temporales han subido del 93 al $99 \%$, mientras que los indefinidos han bajado del 7 al $1 \%{ }^{(13)}$. 
Linda H. Aiken publicó estudios sobre los denominados "hospitales magnéticos" (14,15), son aquellos que atraen a los mejores profesionales médicos y de enfermería. En ellos demostró que los recursos humanos enfermeros tienen influencia en la calidad de los resultados de estos hospitales, en aspectos tan relevantes como la mortalidad o la desatención a las llamadas de pacientes, además de en el propio bienestar de las enfermeras. En hospitales con una relación de 8 ingresados por enfermera, los pacientes tenían un $31 \%$ más de probabilidad de morir que aquellos pacientes de hospitales donde la relación era de 4 pacientes/enfermera. Si aumentara un 10\% la cantidad de enfermeras en los centros sanitarios, se reduciría un $6 \%$ la mortalidad entre los pacientes ${ }^{(15)}$.

El ratio enfermera/paciente ideal sería 1:6. Cuando no existe el número adecuado de enfermeros profesionales en relación con la cantidad de habitantes y pacientes, crecen los eventos adversos y de muerte no relacionados con la causa del ingreso (caídas, infecciones, errores de medicación y quejas) (14).

La Organización de las Naciones Unidas para la Educación, la Ciencia y la Cultura (UNESCO) establece que por cada 1000 habitantes debe haber 7 enfermeras. Teniendo en cuenta que en España actualmente la relación de enfermeras por cada 1000 habitantes es de 5 , cuando debería ser de 7 , con lo que se objetiva respecto al año 2007 una disminución del ratio enfermera/paciente ${ }^{(16)}$.

Enfermería se encuentra dentro de las profesiones de ayuda y las exigencias psicológicas de la actividad laboral incluyen la exposición a emociones y sentimientos humanos, algo especialmente relevante para todos aquellos empleos que conllevan el trabajo para y con usuarios y clientes, y que la investigación ha puesto de manifiesto en las últimas décadas especialmente en relación al síndrome de estar quemado o burnout ${ }^{(17)}$.

La aportación operativa de Maslach y Jackson (1981) definiendo el "burnout" mediante su cuestionario "Maslach Burnout Inventory" (MBI) como un síndrome caracterizado por el cansancio emocional, la despersonalización y la falta de realización personal y profesional, insistía en los mismos elementos y proporcionaba un instrumento que permitía evaluar los niveles del problema ${ }^{(18,19) \text {. }}$

Las condiciones laborales y las características del trabajo desarrollado por el personal de enfermería hacen que el síndrome de burnout tenga una prevalencia alta en esta profesión, estimándose que hasta un $25 \%$ de los profesionales de enfermería estarían afectados por él, con una destacable incidencia en servicios de urgencias, UCI y áreas de oncología ${ }^{(20)}$.

Según el proyecto RN4CAST (Nurse Forecasting In Europe) un $22 \%$ de las enfermeras (1.091) padece un alto burnout en términos globales y un $48 \%$ (2.395) bajo. En cuanto a las dimensiones del Maslach Burnout Inventory cabe destacar que para la de «agotamiento emocional» un 51\% (2.735) de las enfermeras mostraron niveles medios/altos de agotamiento; en la dimensión «despersonalización» un 55\% (3.007) tenían niveles bajos de agotamiento; finalmente, en términos de «realización personal», el nivel de agotamiento medio/alto se presentó en el 3.261 (62\%) de las enfermeras ${ }^{(21)}$.

Para nuestro medio, los datos recogidos en un estudio publicado en el año 2000 entre los profesionales de Servicios de Urgencias del área sanitaria de Albacete y Hellín, 
arrojan los siguientes datos: CE 46,8\%, DP 41,8\%, RP 43\%. Los profesionales con mayor antigüedad (más de 10 años) en su trabajo presentan menos burnout ${ }^{(22)}$.

Los principales desencadenantes del burnout, señalados por la investigación, consisten en la influencia de agentes relacionados con el marco social, el contexto laboral y características personales de los trabajadores. Algunas características o variables de tipo demográfico como la edad, el sexo, el estado civil, y otras condiciones como el clima laboral, la profesión, el nivel de formación y la ambigüedad del rol, entre otras, han sido consideradas como variables que cumplen la función de antecedentes en el desarrollo del síndrome ${ }^{(23)}$.

Dentro del listado de ACV (Acontecimientos Vitales Estresantes) las crisis y problemas económicos figuran como una de las causas más frecuentes que pueden precipitar una respuesta de estrés en el individuo. La crisis económica se está manteniendo lo suficientemente en el tiempo como para que la respuesta de estrés implícita a ella afecte a nuestro organismo, generando problemas de salud física, además de consecuencias en nuestra salud mental y relaciones sociales ${ }^{(24)}$.

Es importante resaltar el rol de las variables de personalidad que sin duda muestran una relación con las respuestas de estrés que manifiestan los individuos ${ }^{(25)}$. La influencia de los factores de personalidad en la etiología del burnout es compleja y multifacética, y probablemente poco explorada. Garden llegó a la conclusión de que ciertos tipos de personalidad auto-seleccionan a los individuos hacia ocupaciones específicas, y que posteriormente los mismos individuos interactúan con ambientes ocupacionales estresantes que son conducentes a la experiencia del burnout (26,27). Kobasa propuso el concepto de personalidad resistente, al observar que algunas personas sometidas a altos niveles de estrés no desarrollan ningún tipo de trastorno de salud. Dos características fundamentales en este modelo son: la conceptualización del hombre como alguien que de forma continua y dinámica va construyendo su personalidad a través de sus conductas, y la consideración de la vida como un inevitable cambio asociado a situaciones de estrés. Este constructo está constituido por tres dimensiones: compromiso, control y reto ${ }^{(28,29)}$. También se ha descubierto que la resistencia, como un rasgo de personalidad, aminora los efectos del estrés en el burnout ${ }^{(30)}$.

La capacidad que tienen las personas de desarrollar mecanismos de superación y afrontamiento, en psicología se denomina resiliencia que se define como "un proceso dinámico que tiene como resultado la adaptación positiva en contextos de adversidad"(31).

Las personas más resilientes mantienen un alto equilibro emocional en momentos de estrés y aguantan bien la presión, lo que les hace sentir que controlan la situación y que son capaces de afrontar los retos. Potenciar la resiliencia es una estrategia para prevenir la ansiedad y la depresión, se trata de aprender a romper el círculo del miedo para superar la adversidad.

Dado que en el contexto de la crisis económica se están produciendo recortes a todos los niveles incluyendo el sanitario, nos parece relevante el estudio de burnout y su relación con las características de personalidad resistente en el personal de enfermería para a su vez como enfermeros especialista en salud mental elaborar posteriormente programas de entrenamiento en habilidades de afrontamiento. 


\section{OBJETIVOS}

\section{Objetivo general}

Describir las condiciones de trabajo, las variables individuales y de salud que están relacionadas con el estrés laboral, así como identificar el nivel de desgaste laboral (burnout), todo esto en el contexto de crisis económica en los profesionales de Enfermería del Complejo Hospitalario Universitario de Albacete y otros organismos sanitarios públicos.

Objetivos específicos:

- Determinar la prevalencia del síndrome de burnout entre los profesionales de Enfermería.

- Describir las puntuaciones de "personalidad resistente/dureza" entre los profesionales de Enfermería, y su distribución según las variables laborales (servicio/unidad).

- Analizar la relación de burnout con los siguientes factores: acontecimientos vitales estresantes, situación laboral y factores relacionados con la crisis económica.

- Determinar si las variables personales consideradas (personalidad resistente) se relacionan con distintos niveles en las tres dimensiones que configuran el síndrome de burnout (agotamiento emocional, despersonalización y falta de realización personal en el trabajo).

- Observar la posible asociación entre burnout y el estado de salud de los profesionales en cuanto a tratamientos farmacológicos de tipo psiquiátrico y/o terapia psicológica que precisen.

\section{METODOLOGÍA}

Estudio observacional, descriptivo, transversal.

Población a estudio: Profesionales de enfermería de Atención Especializada y Atención Sociosanitaria del Sistema Sanitario Público (SESCAM y otros organismos públicos) de la ciudad de Albacete.

Criterios de inclusión: Profesionales de enfermería (Enfermeros y Auxiliares) en activo en el Complejo Hospitalario Universitario de Albacete y centros adscritos.

Criterios de exclusión:

- Profesionales que trabajen en Servicios Centrales (Banco de sangre, Farmacología clínica y Laboratorios, Medicina preventiva, Neurofisiología clínica y Radiodiagnóstico) ya que según la cartera de servicios del CHUA no prestan actividad asistencial directa.

- Profesionales que se encuentren en incapacidad temporal durante el periodo de recogida de datos.

- Profesionales que no firmen el consentimiento informado. 
El cálculo del tamaño muestral se realiza con el programa informático Epi Info 5.0. Para un tamaño de población de 1500 profesionales de enfermería se espera una frecuencia de burnout de $30 \%$ con precisión $4 \%$. Aplicando un intervalo de confianza del 95\% el tamaño muestral es de 377 participantes. Los sujetos se seleccionarán de forma no aleatoria según disponibilidad en servicio/unidad de trabajo.

Variable independiente principal: Condiciones laborales medidas mediante categoría profesional (enfermera o auxiliar de enfermería), experiencia profesional en tiempo trabajado, servicio/unidad de trabajo, antigüedad laboral en actual servicio en tiempo, tipo de contrato (jornada completa/parcial, fijo, interino o eventual), turno de trabajo (fijo de mañanas/tardes/noches, rotatorio), salario mensual actual en euros. Mediante escala tipo Likert se valora empeoramiento de condiciones de trabajo a consecuencia de los recortes y percepción personal del ambiente laboral

Variable independiente moderadora: Acontecimientos vitales estresantes con escala validada de "Acontecimientos Vitales Estresantes" de Holmes y Rahe. Personalidad resistente con escala validada "Cuestionario de Personalidad Resistente" (CPR).

Variable dependiente: Sindrome de Burnout medido con escala validada "Inventario de burnout de Maslach". Tratamiento psicológico y/o psiquiátrico valorado por la toma de medicación psiquiátrica (ansiolíticos, antidepresivos, hipnóticos y/o neurolépticos) especificando nombre comercial del fármaco y/o principio activo, y si reciben terapia psicológica.

Variables sociodemográficas: Sexo, edad en años cumplidos, estado civil, convivencia habitual indicando número de residentes en el domicilio, número de hijos.

En cuanto al sistema de recogida de datos, los participantes responderán de forma individual un cuestionario anónimo y autoadministrado. Se realizará la recogida de datos en los meses de enero y febrero de 2014.

Análisis estadístico:

- Análisis descriptivo de las distintas variables incluidas en el estudio, utilizando frecuencias absolutas y relativas para las variables cualitativas y medidas de tendencia central y dispersión para las cuantitativas. Se calcularán los correspondientes intervalos de confianza al 95\%. Se utilizarán las representaciones gráficas adecuadas para exponer los resultados obtenidos.

- Análisis bivariante, en el que se compararán las posibles asociaciones entre variables teóricamente relacionadas y la existencia de burnout en los profesionales utilizando t de Student o Chi-cuadrado, según la naturaleza de las variables consideradas. Se emplearán otros test no paramétricos si es necesario. Se calcularán las posibles correlaciones utilizando el coeficiente de Pearson.

- Análisis multivariante utilizando regresión logística binaria, en la que la variable dependiente será el burnout y las independientes aquellas que hayan mostrado significación en los análisis bivariados. Para todos los factores incluidos en el modelo se calcularán las Odds ratios (OR) con un 95\% de IC.

El nivel de significación se establecerá para valores de $p<0,05$. Se utilizará IBM SPSS versión 19.0 para la realización de todo el análisis estadístico previsto. 
Limitaciones y sesgos: Destacar que este estudio se realiza con una muestra de profesionales seleccionada de forma no aleatoria, participando los mismos de manera voluntaria y anónima. Esto puede comprometer la generalización de los resultados obtenidos. La recogida de datos se realiza en periodo no vacacional por lo que se prevé que no habrá personal eventual.

Una limitación añadida al diseño del estudio reside en el hecho en que éste únicamente se basa mayoritariamente en la utilización de medidas de autoinforme, con la posible no respuesta. Para tratar de mejorar la participación se harán recordatorios y se motivará al personal. Los cuestionarios de autoinforme son instrumentos validados utilizados en otros estudios con evidencia científica. Un sesgo importante es la imposibilidad de valorar a las enfermeras con incapacidad laboral temporal por no encontrarse presentes en su servicio/unidad.

\section{RESULTADOS}

Variables sociodemográficas: El porcentaje de respuesta de profesionales de enfermería del CHUA fue de 33,54\%. De este el 35,88\% corresponde a hospitalización, el $36,30 \%$ a servicios especiales y el 19,01\% a consultas externas.

La media de edad fue 45,17 años (DT 9.73). El rango de edad estaría comprendido entre 22 y 63 años. En cuanto al sexo el $89.4 \%(n=288)$ fueron mujeres.

La media de personas que conviven en domicilio fueron 2,92(DT=1.20). El número de personas, más frecuente, que convivían en domicilio fue 4. La media de número de hijos fue 1,3 (DT 1.07). El 39.4\% $(n=127)$ de la muestra tiene 2 hijos, un $31.4 \%$ ninguno $(n=101)$. De un total de 245 sujetos con pareja incluidos en la muestra total, el $85.7 \%$ trabaja su pareja mientras que el $14.3 \%$ no trabaja. La media del tiempo de la pareja en paro fue 23.6 meses.

Variables laborales: con respecto a la categoría profesional el $38.4 \%$ eran auxiliares y el $61.6 \%$ enfermeras/os. Repartidos entre un $98.1 \%$ pertenecientes al Servicio de Salud de Castilla La-Mancha (SESCAM) y 1.9\% a la Diputación de Albacete.

La experiencia profesional se estableció en 20.56 (DT 9.94) años de media, mientras que la antigüedad por servicio era 8.03 (DT 8.62) años.

En los tipos de contrato obtenemos un $79.2 \%$ con plaza fija, un $4.4 \%$ de interinos y $16.4 \%$ eventuales, de estos últimos un $42.6 \%$ fue anteriormente interino.

Un 94\% tiene jornada completa y el $6 \%$ parcial. La mayoría presenta un turno rotatorio (81.2\%), fijo de mañanas (18.5\%) y fijo de tardes $(0.3 \%)$.

El salario mensual se agrupó en cinco intervalos:

- Menos de 1000 euros 7\% $(n=22)$

- Entre 1000-1499 euros 36.7\% $(n=116)$

- Entre 1500-1999 euros 26.6\% $(n=84)$

- Entre 2000-2500 euros 28.8\% $(n=91)$

- Más de 2500 euros $0.9 \%(n=3)$

La media de disminución de salario mensual en el último año fue 272.24 euros, estando comprendidos los valores entre 0 y 1000 euros (DT 164.28). A un 3.8\% no le disminuyó el salario. 
Un 15,9\% $(\mathrm{n}=50)$ toma psicofármacos y/o realiza terapia psicológica, dentro de este porcentaje un $44 \%$ tiene tratamiento ansiolítico.

Al analizar el cuestionario sobre condiciones laborales y recortes debidos a la crisis económica observamos que el un 70,2\% $(n=224)$ considera que frecuentemente/siempre ha empeorado las condiciones de trabajo en los últimos meses. En torno al $46.7 \% \quad(n=149)$ considera que frecuentemente/siempre ha empeorado la atención prestada en la unidad de trabajo. El 14,5\% $(n=46)$ opina que nunca existe personal suficiente para prestar una atención adecuada $y$, el $20,8 \%(n=66)$ que raramente. A veces, hasta un $46 \%(n=145)$ le quedan actividades por realizar al acabar el turno. El 44\% $(n=140)$ de la muestra ha sido desplazada a otras unidades que no domina por falta de personal en ellas. La mayoría no ha presentado bajas laborales ligadas a la actividad asistencial $82,5 \% \quad(n=260)$. Impresiona que un $17,6 \%(n=56)$ tenga problemas para hacer frente a los pagos habituales mensuales durante el último año. Finalmente la valoración general sobre el ambiente laboral con una escala Likert que puntúa de 1 a 5 puntos, arroja un $48.1 \%$ $(n=133)$ lo considera regular y el $37.7 \%(n=120)$ bueno.

Los resultados de burnout se muestran en la tabla 1 y 2 . Las dimensiones de le personalidad resistente están representadas en la tabla 3.

Tabla 1. Dimensiones de Burnout $(n=322)$

\begin{tabular}{|l|l|l|l|l|}
\hline & Media & DT & IC 95\% & Rango \\
\hline CE & 23,06 & 12,06 & {$[21.72,24.41]$} & $0-54$ \\
\hline DP & 6,15 & 5,23 & {$[5.57,6.73]$} & $0-26$ \\
\hline RP & 35,33 & 8,20 & {$[34.41,36.52]$} & $0-48$ \\
\hline
\end{tabular}

DT: Desviación típica; IC: Intervalo de confianza; CE: Cansancio emocional; DP: Despersonalización; RP: Realización personal

Tabla 2. Dimensiones de Burnout por niveles $(n=322)$

\begin{tabular}{|l|l|l|l|l|l|l|l|l|}
\hline & \multicolumn{2}{l}{$\begin{array}{l}\text { Cansancio } \\
\text { emocional }\end{array}$} & \multicolumn{2}{l|}{$\begin{array}{l}\text { Gealización } \\
\text { personal }\end{array}$} \\
\cline { 2 - 10 } & $\mathrm{n}$ & $\%$ & $\mathrm{n}$ & $\%$ & $\mathrm{n}$ & $\%$ & $\mathrm{n}$ & $\%$ \\
\hline Bajo & 123 & 38.2 & 156 & 48.4 & 102 & 31.7 & 126 & 39.1 \\
\hline Medio & 74 & 23 & 91 & 28.3 & 100 & 31.1 & 106 & 34.4 \\
\hline Alto & 113 & 35.1 & 67 & 20.8 & 105 & 32.6 & 76 & 23.6 \\
\hline Total & 310 & 96.3 & 314 & 97.5 & 307 & 95.4 & 308 & 97.1 \\
\hline
\end{tabular}

Tabla 3. Dimensiones de CPR ( $n=322)$

\begin{tabular}{|l|l|l|l|l|}
\hline & Media & DT & IC 95\% & Rango \\
\hline Compromiso & 2,92 & 0,47 & {$[2.87,2.97]$} & $1-4$ \\
\hline Control & 1,92 & 0,60 & {$[1.85,1.98]$} & $1-3,67$ \\
\hline Reto & 2,98 & 0,52 & {$[2.93,3.04]$} & $1-4$ \\
\hline CPR total & 2,61 & 0,29 & {$[2.58,2.64]$} & $1,59-3,56$ \\
\hline
\end{tabular}

CPR: Cuestionario de personalidad resistente; DT: Desviación típica; IC: Intervalo de confianza

La variable media de AVE (Acontecimientos vitales estresantes) presentaba valores comprendidos entre 0 y 511 con cierta asimetría positiva $(0,87)$ y aplanamiento (curtosis=0,54). El valor medio de AVE fue de 133,99 (desviación típica=100,50).

Dentro del análisis de la escala de AVE las variables laborables más destacadas son el paro $10,9 \%(n=35)$, el cambio de la situación económica 34,2\% $(n=110)$, cambio de tipo de trabajo $26,4 \%(n=85)$, cambio de responsabilidad del trabajo $8,7 \%(n=28)$ y el cambio de turno o condiciones laborales $41,9 \%(n=135)$. En cuanto a las variables de tipo personal los resultados más prevalentes son la muerte de un familiar cercano $18,3 \%(n=59)$, cambio de salud de un miembro de la familia $38,5 \%(n=124)$, cambio 
en las condiciones de vida $15,2 \% \quad(n=49)$, revisión de hábitos personales $12,6 \%$ $(n=41)$, cambio de actividad de ocio $8,7 \%(n=28)$, cambio de actividades sociales $10,2 \%(n=33)$, cambio de hábitos de sueño $28,9 \%(n=93)$, cambio en el número de reuniones familiares $12,2 \%(n=40)$ y cambio de hábitos alimentarios $12,1 \% \quad(n=39)$. También resulta llamativo la consideración de condición estresante las vacaciones $24,8 \%(n=82)$ y navidades $23 \%(n=74)$.

Las puntuaciones de "personalidad resistente/dureza" entre los profesionales de Enfermería y su distribución por áreas de trabajo están representadas en la tabla 4.

Tabla 4. CPR por áreas de trabajo $(n=322)$

\begin{tabular}{|l|l|l|l|l|}
\hline & Media & DT & IC 95\% & Rango \\
\hline Hospitalización & 2.57 & 0.29 & {$[2.52,2.61]$} & $1.59-3.38$ \\
\hline Servicios especiales & 2.66 & 0.26 & {$[2.61,2.71]$} & $1.89-3.47$ \\
\hline Consultas & 2.72 & 0.34 & {$[2.59,2.86]$} & $2.27-3.56$ \\
\hline
\end{tabular}

CPR: Cuestionario de personalidad resistente; DT: Desviación típica; IC: Intervalo de confianza

Analizando la relación entre dimensiones de burnout y variables sociodemográficas encontramos un mayor cansancio emocional en mujeres respecto a hombres $(\mathrm{t}=-2.17$; $\mathrm{p}=0.031$ ), siendo la media para mujeres de 23.51 y hombres de 18.58 ; y puntuaciones más elevadas de despersonalización ( $\mathrm{t}=-2.91 ; \mathrm{p}=0.04)$ y menor realización personal en turno rotatorio en comparación a turno fijo $(t=2.77 ; p=0.06)$. En la comparación por áreas de trabajo observamos que los trabajadores de servicios especiales muestran mayor despersonalización $\left(X^{2}=7.63 ; p=0.022\right)$ y los de consultas mayor realización personal $\left(X^{2}=6.08 ; p=0.048\right)$.

Tabla 5. Relación entre burnout y variables sociodemográficas $(n=322)$

\begin{tabular}{|c|c|c|c|c|c|c|}
\hline \multicolumn{2}{|c|}{ VARIABLES SOCIODEMOGRÁFICAS } & \multirow{2}{*}{$\begin{array}{l}\text { Burnout } \\
\text { alto } n(\%)\end{array}$} & \multirow{2}{*}{$\begin{array}{l}\text { Burnout } \\
\text { medio/bajo } \\
\text { n(\%) } \\
24(7.8)\end{array}$} & \multirow{2}{*}{$\begin{array}{l}\mathbf{X}^{2} \\
0.35\end{array}$} & \multirow{2}{*}{$\begin{array}{l}\text { OR } \\
0.75\end{array}$} & \multirow{2}{*}{$\begin{array}{l}\text { IC 95\% } \\
{[0.26,2.05]}\end{array}$} \\
\hline \multirow[t]{2}{*}{ Sexo } & Hombre & & & & & \\
\hline & Mujer & $69(22.5)$ & $208(67.8)$ & & & \\
\hline \multirow[t]{2}{*}{ Categoría profesional } & Auxiliar & $30(9.8)$ & $88(28.7)$ & \multirow[t]{2}{*}{0.10} & \multirow[t]{2}{*}{1.09} & \multirow[t]{2}{*}[0.62,1.92]{} \\
\hline & Enfermera & $45(14.7)$ & 144(46.9) & & & \\
\hline \multirow[t]{2}{*}{ Jornada trabajo } & Completa & $69(22.6)$ & $217(71.1)$ & \multirow[t]{2}{*}{0.53} & \multirow[t]{2}{*}{0.69} & \multirow[t]{2}{*}[0.23,2.12]{} \\
\hline & Parcial & $6(2)$ & $13(4.3)$ & & & \\
\hline \multirow[t]{2}{*}{ Turno trabajo } & Fijo & $12(3.9)$ & $46(15)$ & \multirow[t]{2}{*}{0.56} & \multirow[t]{2}{*}{0.77} & \multirow[t]{2}{*}[0.36,1.61]{} \\
\hline & Rotatorio & $63(20.6)$ & $185(60.5)$ & & & \\
\hline \multirow[t]{2}{*}{ Tratamiento PF } & Sí & $19(6.3)$ & $30(9.9)$ & \multirow[t]{2}{*}{6.17} & \multirow[t]{2}{*}{2.24} & \multirow[t]{2}{*}[1.12,4.48]{} \\
\hline & No & $56(18.5)$ & 198(65.3) & & & \\
\hline
\end{tabular}

$\mathrm{X}^{2}$ : chi cuadrado; OR: Odds ratio; IC: Intervalo de confianza; PF: Psicofármacos

La despersonalización se correlaciona de manera directa con la disminución del salario (Pearson $=0,117, p=0.040$ ) y con la puntuación de acontecimientos vitales estresantes (Pearson=0.273, $\mathrm{p}=0.001$ ) y de manera inversa con los años de antigüedad en el servicio actual (Pearson=-0.132, $\mathrm{p}=0.02$ ).

En el análisis entre las áreas de trabajo y la valoración del ambiente laboral la diferencia es estadísticamente significativa $\left(X^{2}=20.534 ; p=0.008\right)$. En una muestra de 315 profesionales, un $41.9 \%$ valora el ambiente laboral como regular y un $37.8 \%$ como bueno.

La relación entre dimensiones de burnout y variables laborales muestra que el cansancio emocional es mayor en aquellos profesionales que valoran el ambiente laboral como malo/muy malo frente a los que lo consideran regular $(p=0.001) 0$ bueno/muy bueno $(p=0.001)$. Al igual ocurre con la despersonalización con similar 
significación $(p=0.001)$ para ambos. En cuanto a realización personal, esta es mayor en aquellos que valoran su ambiente laboral como bueno/muy bueno frente a los que lo valoran como malo/muy malo $(p=0.026)$

En el empeoramiento en las condiciones de trabajo y de la atención prestada se observó que los que consideran que ha empeorado frecuentemente/siempre presentan mayor cansancio emocional que lo que lo consideran nunca/raramente/a veces $(t=-7.006, \quad p=0.001)$. De forma similar ocurre con la dimensión despersonalización $(\mathrm{t}=-5.861, \mathrm{p}=0.001)$.

Quienes tienen pendientes actividades al terminar su turno de trabajo frecuentemente/siempre presentan un mayor cansancio emocional $(t=-3.054, p=$ 0.002). Al igual ocurre con los que tienen que aumentar su jornada de trabajo ( $\mathrm{t}=-$ 2.927, $\mathrm{p}=0.004)$, los que han sido desplazados a otras unidades de trabajo $(\mathrm{t}=-2.89$, $\mathrm{p}=0.004)$ y aquellos que tienen dificultades en sus pagos mensuales $(\mathrm{t}=-2.843, \mathrm{p}=$ $0.005)$.

Existe además correlación directa entre la puntuación de acontecimientos vitales estresantes y cansancio emocional (Pearson=0.134, $\mathrm{p}=0.018$ )

En el estudio de las dimensiones de burnout frente a los acontecimientos vitales estresantes, y según significación estadística, encontramos que los profesionales que han experimentado cambio de turno o condiciones laborales presentan mayor cansancio emocional frente a los que no $(\mathrm{t}=-2.232, \mathrm{p}=0.026)$. Ocurre del mismo modo en aquellos que han tenido una muerte de un familiar cercano $(t=-2128, p=0.034)$, los que tienen un cambio en su situación económica ( $\mathrm{t}=-2.202, \mathrm{p}=0.028)$, en los que han cambiado las condiciones de trabajo $(\mathrm{t}=-2.462, \mathrm{p}=0.014)$, cambio de actividades sociales $(\mathrm{t}=-2.681, \mathrm{p}=0.008)$ y cambio de hábitos alimentarios $(\mathrm{t}=-3.087, \mathrm{p}=0.002)$. Los que han sufrido un cambio del hábito de sueño presentaron mayor cansancio emocional $(t=-5.020, p=0.001)$ y despersonalización $(t=-2.674, p=0.008)$.

Al correlacionar las dimensiones de burnout con el cuestionario de personalidad resistente obtenemos una correlación directa entre despersonalización (Pearson=0.151; $p=0.008$ ) y realización personal (Pearson $=0.175 ; p=0.002$ ).

Hay significación estadística entre la resilencia y el estado civil (Kruskal-Wallis, $\mathrm{X}^{2}=$ $11,543, p=0,021)$.

\section{DISCUSIÓN}

El propósito de esta investigación fue describir las relaciones existentes entre el síndrome de quemarse por el trabajo, evaluado con la versión española del $\mathrm{MBI}$, la personalidad resistente, evaluada con la escala CPR, y la frecuencia de acontecimientos vitales estresantes en el personal de enfermería, valorada mediante la escala de acontecimientos vitales estresantes de Holmes y Rahe. Pretendíamos también explorar las variables sociodemográficas y laborales.

Nuestro porcentaje de participación (33.54\%) permite considerar relativamente representativa la población diana que se pretendía estudiar. Esta tasa de respuesta es menor a la obtenida por otros estudios de nuestro entorno sociocultural y laboral ${ }^{[21,29] \text {. }}$ 
La media de edad de los participantes corresponde a la media de edad de la muestra a estudio, siendo mayor a la de otros estudios al respecto ${ }^{[19,21,29]}$ datos que se contrastan con que el porcentaje de profesionales con contrato eventual (16.4\%) es menor, del mismo modo, al recogido en otros estudios [11]. Casi el $80 \%$ tiene plaza fija.

El sexo de los participantes mantiene la proporcionalidad existente en la profesión enfermera, con un mayor número de mujeres. Los participantes presentan un tiempo de trabajo estable (20.56 años) y elevada media de años en los servicios (8.03 años), tiempo suficiente para permitir comparar situaciones anteriores de bonanza económica con la actual de crisis.

La prevalencia del síndrome de burnout obtenida en la muestra estudiada nos muestra un nivel moderado-alto, siendo especialmente relevante la alta tasa de CE, que refiere un $35.1 \%$ de los encuestados, tal y como se observa en la tabla 12. También destaca el nivel bajo de RP, que refiere el $32.6 \%$ de la muestra. En cuanto a la dimensión despersonalización un $20.8 \%$ presenta altos niveles. Estos resultados vienen a ser similares a los encontrados en el proyecto RN4CAST-España [21] y menores a los obtenidos por Rios-Risquez ${ }^{[29]}$ en Murcia y de la Secretaría general de SATSE ${ }^{[11]}$, o algo superiores a los obtenidos por algunos estudios que analizan el síndrome de burnout en el personal sanitario ${ }^{[22]}$. Además es coincidente con estudios previos en la dimensión de CE, la que aglutina un peso más relevante entre las tres dimensiones que configuran el cuadro sintomático del burnout.

Tabla 6. Comparación de dimensiones de burnout con otros estudios

\begin{tabular}{|l|c|c|c|c|}
\hline & $\mathbf{C E}(\%)$ & $\mathbf{D P}(\%)$ & $\mathbf{R P}(\%)$ & Total(\%) \\
\hline Nuestra muestra & 35.1 & 20.8 & 32.6 & 23.6 \\
\hline RN4CAST & 26.25 & 22.24 & 34.24 & 22 \\
\hline Rios-Risquez & 54.8 & 35.7 & 21.4 & 11.9 \\
\hline SATSE & 62 & 77 & No datos & 62 \\
\hline
\end{tabular}

CE: Cansancio emocional; DP: Despersonalización; RP: Realización personal; RN4CAST:

Nurse forecasting in Europe; SATSE: Sindicato de enfermería

Por lo que respecta al análisis del perfil de riesgo para padecer burnout, coincidimos con multitud de estudios previos que expresan la falta de consenso. En nuestra muestra utilizamos metodología similar a la usada en RN4CAST, siendo distinta al estudio de Rios-Risquez y de la Secretaría general de SATSE, que utiliza un cuestionario de elaboración propia para medir burnout.

Al analizar las condiciones laborales y recortes debidos a la crisis económica observamos que un $70,2 \%$ considera que frecuentemente/siempre han empeorado las condiciones de trabajo en los últimos meses. En torno al $46.7 \%$ considera que frecuentemente/siempre ha empeorado la atención prestada en la unidad de trabajo. El $14,5 \%$ opina que nunca existe personal suficiente para prestar una atención adecuada y, el $20,8 \%$ que raramente. A veces, hasta un $46 \%$ le quedan actividades por realizar al acabar el turno. El $44 \%$ de la muestra ha sido desplazada a otras unidades que no domina por falta de personal en ellas. La mayoría no ha presentado bajas laborales ligadas a la actividad asistencial (82,5\%). Impresiona que un 17,6\% tenga problemas para hacer frente a los pagos habituales mensuales durante el último año. Finalmente la valoración general sobre el ambiente laboral con una escala Likert que puntúa de 1 a 5 puntos, arroja un $48.1 \%$ lo considera regular y el $37.7 \%$ bueno. 
Si comparamos nuestros resultados con los obtenidos por el estudio de la Secretaría general de SATSE encontramos resultados similares y menores en algunas de las variables estudiadas, como muestra la tabla 7.

Tabla 7. Comparación de condiciones laborales y recortes debidos a la crisis económica respecto al estudio realizacido por la secretaría general del SATSE

\begin{tabular}{|c|c|c|}
\hline & Nuestra muestra & SATSE \\
\hline Empeoramiento condiciones de trabajo & $\begin{array}{c}70.2 \% \\
\text { frecuentemente/siempre }\end{array}$ & $88 \%$ deteriorado \\
\hline $\begin{array}{l}\text { Empeoramiento de la atención prestada en la } \\
\text { unidad }\end{array}$ & $\begin{array}{c}46.7 \% \\
\text { frecuentemente/siempre }\end{array}$ & $51 \%$ \\
\hline $\begin{array}{l}\text { Existencia de personal suficiente para prestar } \\
\text { atención adecuada }\end{array}$ & $20.8 \%$ raramente & $\begin{array}{l}80 \% \text { no hay adecuado } \\
\text { personal }\end{array}$ \\
\hline Actividades por realizar al acabar el turno & $46 \%$ & $65 \%$ \\
\hline $\begin{array}{l}\text { Prolongación del turno para realizar actividades } \\
\text { pendientes }\end{array}$ & $17 \%$ frecuentemente/siempre & $59 \%$ \\
\hline Desplazamiento a otras unidades sin dominio & $44 \%$ & $49 \%$ \\
\hline $\begin{array}{l}\text { Bajas laborales por problemas psicológicos } \\
\text { ligadas a la actividad laboral }\end{array}$ & $1.6 \%$ frecuentemente & $9 \%$ \\
\hline Valoración ambiente laboral & $48.1 \%$ regular & $86 \%$ deteriorado \\
\hline
\end{tabular}

Dada la escasez de estudios que han examinado la relación entre la resilencia o personalidad resistente como variable protectora y el tipo de variables consideradas, las comparaciones con otros trabajos previos se ven limitadas en la discusión de los resultados obtenidos para este objetivo. La gran mayoría de publicaciones que se han centrado en el estudio del síndrome de quemarse por el trabajo y sus consecuencias asociadas, han enfatizado el papel desempeñado por los estresores y variables organizacionales, quedando al margen la influencia de variables de tipo individual. Sin embargo, queda demostrado que estas variables personales deben ser tomadas en consideración. La forma en que las personas perciben, sienten y actúan (su personalidad) media o modula las consecuencias que un ambiente laboral estresante pueda tener sobre la persona. Este aspecto resulta especialmente relevante en el caso de los profesionales de enfermería, frecuentemente sometidos a severas fuentes de estrés como son el afrontamiento del dolor, la enfermedad, el sufrimiento, la muerte, la incertidumbre, etc.

En este sentido, encontramos en nuestro estudio que aquellos individuos que expresan mayores niveles de personalidad resistente experimentan menos el síndrome de quemarse por el trabajo, así como una menor frecuencia de síntomas de malestar psíquico.

Los datos obtenidos en nuestro estudio con respecto a la personalidad resistente son similares a los obtenidos en el estudio realizado por Ríos Risquez ${ }^{[29]}$ siendo ligeramente menor la dimensión control en nuestro estudio.

Con la presente investigación se ha pretendido profundizar en la relevancia que determinadas variables de tipo individual pueden tener a la hora de explicar el desarrollo y mantenimiento del síndrome de quemarse por el trabajo y la frecuencia de sintomatología psíquica relacionada con el mismo. Es destacable la prevalencia de tratamiento con psicofármacos y/o realiza terapia psicológica, de un 15,9\%, de los cuales un $44 \%$ tiene tratamiento ansiolítico. 
También hemos analizado la importancia de los acontecimientos vitales estresantes. Dentro del análisis de la escala de AVE las variables laborables más destacadas son el paro $(10,9 \%)$, el cambio de la situación económica $(34,2 \%)$, cambio de tipo de trabajo $(26,4 \%)$, cambio de responsabilidad del trabajo $(8,7 \%)$ y el cambio de turno 0 condiciones laborales(41,9\%). En cuanto a las variables de tipo personal los resultados más prevalentes son la muerte de un familiar cercano (18,3\%), cambio de salud de un miembro de la familia(38,5\%), cambio en las condiciones de vida(15,2\%), revisión de hábitos personales(12,6\%), cambio de actividad de ocio(8,7\%), cambio de actividades sociales $(10,2 \%)$, cambio de hábitos de sueño(28,9\%), cambio en el número de reuniones familiares(12,2\%) y cambio de hábitos alimentarios(12,1\%). También resulta llamativo la consideración de condición estresante las vacaciones $(24,8 \%)$ y navidades $(23 \%)$.

\section{CONCLUSIONES}

En nuestro estudio se observa que el síndrome de burnout es ligeramente superior al periodo precrisis. El cansancio emocional es la dimensión más influyente en la aparición del síndrome y aquella que se ha visto más alterada desde el inicio de la crisis económica.

Aquellos factores que predisponen al aumento de burnout son el sexo femenino, turno rotario de trabajo, desarrollo profesional en servicios especiales, pocos años de experiencia en el servicio/unidad y la valoración del ambiente laboral precario.

Los profesionales advierten un empeoramiento de las condiciones laborales, viéndose deteriorada la atención prestada a los pacientes. Esto es percibido a modo de falta de personal suficiente, sobrecarga laboral, disminución de tiempo para la atención directa con el paciente, aumento de la jornada laboral, falta de solidaridad con los profesionales por parte de los gestores. La disminución salarial unida a otros condicionantes personales dificulta el afrontamiento de los pagos mensuales habituales lo que se configura como otra de las variables que influye en el desarrollo del síndrome de burnout.

En cuanto a los acontecimientos vitales estresantes, son una circunstancia ligada a la aparición del síndrome de burnout sin poder determinar si son desencadenantes o son producidos por la clínica de éste. Aquellos detectados más frecuentemente son los relacionados con la actividad laboral, familiar y económica.

Tanto el tratamiento psicofarmacológico/psicológico como los cambios en los hábitos de dormir son factores de riesgo en la aparición de burnout.

La importancia del constructo de la personalidad resistente en la problemática del síndrome de quemarse por el trabajo también viene dada por su integración en las posibles medidas de prevención y tratamiento. Se evidencia, pues, el potencial protector de esta estructura de la personalidad. Una pieza clave en este estudio es la correlación inversa entre burnout y resiliencia, de modo que cuando aumenta una disminuye la otra. Con lo que podemos concluir que medidas para favorecer/mejorar la resilencia (ha demostrado ser modulable) previenen la aparición del síndrome. Futuras líneas de investigación podrían estar encaminadas al estudio de medidas de mejora de resilencia como técnicas y programas dirigidos a fomentar el afrontamiento adaptativo personal, entrenamiento en solución de problemas, asertividad, manejo 
eficaz del tiempo, desarrollo de habilidades sociales, manejo de la autoestima, etc; trabajo específico de la enfermería de Salud Mental.

\section{REFERENCIAS}

1. Torrero A. A propósito de la crisis financiera actual. Ekonomiaz 2007; 66(3): 8585.

2. Dávila CD, López-Valcárcel BG. Crisis económica y salud. Gaceta Sanitaria 2009; 23(4): 261-265.

3. Zabalegui A, Cabrera E. Economic crisis and nursing in Spain. Journal of Nursing Management 2010; 18: 505-508.

4. Sermeus W. Impact of the financial crisis and health workforce crisis on the sustainability of the healthcare system: what's next? Enferm Clin 2012; 22 (4): 179181.

5. López-Fernández LA et al. ¿Está en peligro la cobertura universal en nuestro Sistema Nacional de Salud?. Gac Sanit 2012; 26(4):298-300.

6. Boletín General del Estado. Real Decreto-Ley 16/2012, de 20 de abril, de medidas urgentes para garantizar la sostenibilidad del Sistema Nacional de Salud y mejorar la calidad y seguridad de sus prestaciones. BOE núm 98, 24/04/2012; Sec. I: 31278-312.

7. Boletín General del Estado. Real Decreto-Ley 20/2012, de 13 de julio, de medidas para garantizar la estabilidad presupuestaria y de fomento de la competitividad. BOE núm. 168 13/07/2012.

8. World Health Organization (WHO). World Health Statistics 2011. Disponible en: www.who.int Visitado: 19/02/2013 13:30 horas.

9. Martínez Riera JR. Crisis y enfermeras. Rev ROL Enf 2012; 35(2):108-119.

10. European Federation of Nurses Associations (EFN). The Impact of the Financial Crisis on Nurses and Nursing. January 2012. Disponible en: http://www.efnweb.be/wpcontent/uploads/2012/05/EFN-Report-on-the-Impact-of-the-Financial-Crisis-on-

Nurses-and-Nursing-January-20122.pdf Visitado: 23/01/2013, 18:34 horas.

11. Sindicato de enfermería (SATSE). Crisis y estrés en profesionales de enfermería. 2012.2 Disponible en: http://www.satse.es/media/documentos/informes/estudio-satse-percepcion-de-estresen-los-profesionales-de-enfermeria-en-espana. Visitado: 23/01/2013, 18:34h.

12. Staiger D, Auerbach D, Buerhaus P. Registered Nurse Labor Supply and the Recession. Are We in a Bubble? New England Journal of Medicine 2012; 366:14631465.

13. Romero G. El desempleo llega a la profesión: 16.375 enfermeros en paro. Enfermería facultativa 2012; 160: 6-9.

14. Aiken LH, Clarke SP, Sloane DM. Hospital staffing, organization, and quality of care: cross-national findings Nurs, Outlook 2002; 50:187-194.

15. Aiken LH, Clarke SP, Cheung RB, Sloane DM, Silber JH. Educational levels of hospital nurses and surgical patient mortality. JAMA 2003; 290:1617-1623.

16. Prieto B, Gómez MP, Sánchez MC, Díaz S. Situación de la Enfermería en Europa. Rev Salud 2000 2007;(114):21.

17. Pomer MC, Giner I. Síndrome de estar quemado (Burnout), un riesgo laboral en aumento. Prevalencia en enfermeras del CHGU de Valencia. Enfermería integral 2011;(94):21-24.

18. Maslach C, Jackson SE. The Maslach Burnout Inventory. Research Edition, Palo Alto (CA): Consulting Psychologist Press 1981. 
19. Moreno B, Gonzalez JL, Garrosa E. Desgaste profesional (burnout), personalidad y salud percibida. Empleo, estrés y salud 2001:59-83.

20. Tomás J, Maynegre M, Pérez M, Alsina M, Quinta R, Granell S. Síndrome de burnout y riesgo suicida en enfermeras de atención primaria. Enferm Clin 2010;20(3):173-178.

21. Fuentelsaz-Gallego C, Moreno T, López D, Gómez T, González E. Percepción del entorno laboral de las enfermeras españolas en los hospitales del Sistema Nacional de Salud. Proyecto RN4CAST-España. Enferm Clin 2012;22(5):261-268.

22. Fagoaga ML, Lucas FJ. El Síndrome de burnout y la enfermería de urgencias. Revista de enfermería 2000;12:6-12.

23. Benavides IG, Gimeno D, Benach C. Descripción de los factores de riesgo psicosocial en cuatro empresas. Gaceta Sanitaria 2002;16(3):222-229.

24. Holmes T, Rahe R. The social readjustment rating scale. J. Psychoson 1967;11:213-218.

25. Avila JH, Gómez LT, Montiel MM. Características demográficas y laborales asociadas al síndrome de burnout en profesionales de la salud. Pensamiento psicológico 2010; 8(15):39-52.

26. Garden AM. Burnout: the effect of psychological type on research findings. Journal of Occupational Psychology 1989;62:223.

27. Garden AM. The purpose of burnout: a Jungian interpretation. J Soc Behav Pers 1991;6:73-93.

28. Kobasa SC. Stressful life events, personality, and health: an inquiry into hardiness. Personality and Social Psychology 1979;37:1-11.

29. Ríos MI, Godoy C, Sánchez-Mecas J. Síndrome de quemarse por el trabajo, personalidad resistente y malestar psicológico en personal de enfermería. Anales de psicología Ene 2011;27(1):71-79.

30. Shirom A. On the construct Validity, predictors and consequences of burnout at work. Cienc Trab Abri-jun 2009;11(32):44-54.

31. Cortés JE. La resiliencia: una mirada desde la enfermería. Cienc Enferm 2010;16(3):27-32. 


\section{ANEXOS}

\section{VARIABLES SOCIO-DEMOGRÁFICAS}

1. Edad (años cumplidos) :

2. Sexo:

$\square$ Hombre

$\square$ Mujer

3. Estado Civil: $\square$ Soltero

$\square$ Casado

$\square$ Viudo

$\square$ Pareja de hecho

Divorciado/separado

4. ¿Cuántas personas viven en tu domicilio habitual?

¿Número de personas

$\square$ Vivo solo/a

5. № de hijos (Ninguno=0) :

6. Categoría profesional: $\square$ Auxiliar de enfermería $\square$ Enfermera/o

7. Organismo donde prestas tu servicio: $\square$ SESCAM $\square$ Diputación de Albacete

8. ¿Trabaja tu pareja? $\square$ Sí $\square$ No (en caso de que la respuesta sea no, cuánto tiempo lleva sin trabajar? años meses días

9. Experiencia profesional: años meses días

10. Servicio en el que trabaja:

11. Antigüedad laboral en el actual servicio: años meses días

12. Tipo de contrato: $\square$ Fijo $\square$ Interino $\square$ Eventual ¿anteriormente tu contrato era de interino? $\square$ Sí $\square$ No

13. Tipo de jornada: $\square$ Jornada completa $\square$ Jornada parcial

14. Turno en el que trabaja: $\square$ Fijo de mañanas $\square$ Fijo de tardes $\square$ Fijo de noches $\square$ Rotatorio.

15. Salario mensual actual: $\square$ Menos 1000 euros $\square$ 1000-1499 euros $\square$ 1500-1999 euros $\square$ 2000-2500 euros $\square$ Más de 2500 euros

16. ¿Cuánto ha disminuido tu salario mensual en el último año? euros.

17. Medicación (ansiolíticos, antidepresivos, hipnóticos y/o neurolépticos) y/o terapia psicológica:

$\square$ No

$\square$ Sí (Si la respuesta es afirmativa especifique nombre del fármaco) 
VARIABLES LABORALES

Rellenar con una cruz sobre la casilla que corresponda:

\begin{tabular}{|c|c|c|c|c|c|c|}
\hline & & Nunca & Raramente & A veces & Frecuentemente & Siempre \\
\hline 1. & $\begin{array}{l}\text { ¿Han empeorado tus condiciones } \\
\text { de trabajo en los últimos meses a } \\
\text { consecuencia de los recortes? }\end{array}$ & 1 & 2 & 3 & 4 & 5 \\
\hline 2. & $\begin{array}{l}\text { ¿Crees que ha empeorado la } \\
\text { atención que se presta en tu } \\
\text { unidad? }\end{array}$ & 1 & 2 & 3 & 4 & 5 \\
\hline 3. & $\begin{array}{l}\text { ¿En tu unidad existe personal } \\
\text { suficiente para prestar la atención } \\
\text { más adecuada? }\end{array}$ & 1 & 2 & 3 & 4 & 5 \\
\hline 4. & $\begin{array}{l}\text { ¿Cuándo acaba tu turno de } \\
\text { trabajo te quedan actividades por } \\
\text { realizar? }\end{array}$ & 1 & 2 & 3 & 4 & 5 \\
\hline 5. & $\begin{array}{l}\text { ¿Prolongas tu turno de trabajo } \\
\text { para realizar actividades que no } \\
\text { has podido realizar en él? }\end{array}$ & 1 & 2 & 3 & 4 & 5 \\
\hline 6. & $\begin{array}{l}\text { ¿Te has visto desplazado a otras } \\
\text { unidades que no dominas por } \\
\text { falta de personal en ellas? }\end{array}$ & 1 & 2 & 3 & 4 & 5 \\
\hline 7. & $\begin{array}{l}\text { ¿Has tenido bajas laborales por } \\
\text { problemas psicológicos ligadas a } \\
\text { la actividad laboral? }\end{array}$ & 1 & 2 & 3 & 4 & 5 \\
\hline \multirow[t]{2}{*}{8.} & $\begin{array}{l}\text { ¿Te resulta más difícil en el } \\
\text { último año hacer frente a los } \\
\text { pagos habituales mensuales a } \\
\text { consecuencia de la disminución } \\
\text { salarial por parte del gobierno? }\end{array}$ & 1 & 2 & 3 & 4 & 5 \\
\hline & & $\begin{array}{l}\text { Muy } \\
\text { malo }\end{array}$ & Malo & $\begin{array}{c}\text { Regula } \\
\mathrm{r}\end{array}$ & Bueno & $\begin{array}{c}\text { Muy } \\
\text { bueno }\end{array}$ \\
\hline 9. & $\begin{array}{l}\text { ¿Cómo valoras tu ambiente } \\
\text { laboral? }\end{array}$ & 1 & 2 & 3 & 4 & 5 \\
\hline
\end{tabular}




\section{MBI (INVENTARIO DE BURNOUT DE MASLACH)}

A cada una de las frases debe responder expresando la frecuencia con que tiene ese sentimiento, poniendo una cruz en la casilla correspondiente que considere más adecuada.

\begin{tabular}{|c|c|c|c|c|c|c|c|c|}
\hline & & Nunca & $\begin{array}{l}\text { Alguna vez } \\
\text { al año o } \\
\text { menos }\end{array}$ & \begin{tabular}{|c|} 
Una vez \\
al mes o \\
menos
\end{tabular} & $\begin{array}{l}\text { Algunas } \\
\text { veces al } \\
\text { mes }\end{array}$ & $\begin{array}{l}\text { Una vez } \\
\text { a la } \\
\text { semana }\end{array}$ & \begin{tabular}{|c|} 
Varias \\
veces a la \\
semana
\end{tabular} & Diariamente \\
\hline & & 0 & 1 & 2 & 3 & 4 & 5 & 6 \\
\hline 1 & $\begin{array}{l}\text { Debido a mi trabajo me siento } \\
\text { emocionalmente agotado. }\end{array}$ & & & & & & & \\
\hline 2 & $\begin{array}{l}\text { Al final de la jornada me siento } \\
\text { agotado. }\end{array}$ & & & & & & & \\
\hline 3 & $\begin{array}{l}\text { Me encuentro cansado cuando } \\
\text { me levanto por las mañanas y } \\
\text { tengo que enfrentarme a otro día } \\
\text { de trabajo. }\end{array}$ & & & & & & & \\
\hline 4 & $\begin{array}{l}\text { Puedo entender con facilidad lo } \\
\text { que piensan mis pacientes. }\end{array}$ & & & & & & & \\
\hline 5 & $\begin{array}{l}\text { Creo que trato a algunos } \\
\text { pacientes como si fueran objetos. }\end{array}$ & & & & & & & \\
\hline 6 & $\begin{array}{l}\text { Trabajar con pacientes todos los } \\
\text { días es una tensión para mí. }\end{array}$ & & & & & & & \\
\hline 7 & $\begin{array}{l}\text { Me enfrento muy bien con los } \\
\text { problemas que me presentan mis } \\
\text { pacientes. }\end{array}$ & & & & & & & \\
\hline 8 & $\begin{array}{l}\text { Me siento "quemado" por el } \\
\text { trabajo. }\end{array}$ & & & & & & & \\
\hline 9 & $\begin{array}{l}\text { Siento que mediante mi trabajo } \\
\text { estoy influyendo positivamente en } \\
\text { la vida de otros. }\end{array}$ & & & & & & & \\
\hline 10 & $\begin{array}{l}\text { Creo que tengo un } \\
\text { comportamiento más insensible } \\
\text { con la gente desde que hago este } \\
\text { trabajo. }\end{array}$ & & & & & & & \\
\hline 11 & $\begin{array}{l}\text { Me preocupa que este trabajo me } \\
\text { esté endureciendo } \\
\text { emocionalmente. }\end{array}$ & & & & & & & \\
\hline 12 & $\begin{array}{l}\text { Me encuentro con mucha } \\
\text { vitalidad. }\end{array}$ & & & & & & & \\
\hline 13 & $\begin{array}{l}\text { Me siento frustrado por mi } \\
\text { trabajo. }\end{array}$ & & & & & & & \\
\hline 14 & $\begin{array}{l}\text { Siento que estoy haciendo un } \\
\text { trabajo demasiado duro. }\end{array}$ & & & & & & & \\
\hline 15 & $\begin{array}{l}\text { Realmente no me importa lo que } \\
\text { les ocurrirá a algunos de los } \\
\text { pacientes a los que tengo que } \\
\text { atender. }\end{array}$ & & & & & & & \\
\hline 16 & $\begin{array}{l}\text { Trabajar en contacto directo con } \\
\text { los pacientes me produce } \\
\text { bastante estrés. }\end{array}$ & & & & & & & \\
\hline 17 & $\begin{array}{l}\text { Tengo facilidad para crear una } \\
\text { atmósfera relajada a mis } \\
\text { pacientes. }\end{array}$ & & & & & & & \\
\hline 18 & $\begin{array}{l}\text { Me encuentro animado después } \\
\text { de trabajar junto con los } \\
\text { pacientes. }\end{array}$ & & & & & & & \\
\hline 19 & $\begin{array}{l}\text { He realizado muchas cosas que } \\
\text { merecen la pena en este trabajo. }\end{array}$ & & & & & & & \\
\hline 20 & $\begin{array}{l}\text { En el trabajo siento que estoy al } \\
\text { límite de mis posibilidades. }\end{array}$ & & & & & & & \\
\hline 21 & $\begin{array}{l}\text { Siento que se tratar de forma } \\
\text { adecuada los problemas } \\
\text { emocionales en el trabajo. }\end{array}$ & & & & & & & \\
\hline 22 & $\begin{array}{l}\text { Siento que los pacientes me } \\
\text { culpan de algunos de sus } \\
\text { problemas. }\end{array}$ & & & & & & & \\
\hline
\end{tabular}




\section{CPR (CUESTIONARIO DE PERSONALIDAD RESILIENTE)}

A continuación encontrará una serie de cuestiones con las que podrá estar de acuerdo o no. Por favor, indique lo que piensa en relación con casa una de ellas, marcando con una cruz el número que mejor describa su opinión según la siguiente escala de respuesta:

\section{$1=$ totalmente en desacuerdo \\ $2=$ en desacuerdo \\ $3=$ de acuerdo \\ $4=$ totalmente de acuerdo}

\begin{tabular}{|c|c|c|c|c|}
\hline 1. Me gusta que haya una gran variedad en mi trabajo & 1 & 2 & 3 & 4 \\
\hline 2. Tengo una gran curiosidad por lo novedoso tanto a nivel personal como profesional & 1 & 2 & 3 & 4 \\
\hline 3. Mi trabajo cotidiano me satisface y hace que me dedique totalmente a él & 1 & 2 & 3 & 4 \\
\hline $\begin{array}{l}\text { 4.Considero que el trabajo que realizo es de valor para la sociedad y no me importa } \\
\text { dedicarle todos mis esfuerzos. }\end{array}$ & 1 & 2 & 3 & 4 \\
\hline $\begin{array}{l}\text { 5. Frecuentemente siento que puedo cambiar lo que podría ocurrir mañana a través de lo } \\
\text { que estoy haciendo hoy. }\end{array}$ & 1 & 2 & 3 & 4 \\
\hline 6. Aunque me esfuerce no se consigue nada & 1 & 2 & 3 & 4 \\
\hline 7. Mis propias ilusiones son las que siga adelante con la realización de mi actividad & 1 & 2 & 3 & 4 \\
\hline 8. Realmente me preocupo y me identifico con mi trabajo & 1 & 2 & 3 & 4 \\
\hline $\begin{array}{l}\text { 9. Aún cuando suponga mayor esfuerzo, opto por los trabajos que suponen para mi una } \\
\text { experiencia nueva }\end{array}$ & 1 & 2 & 3 & 4 \\
\hline $\begin{array}{l}\text { 10. En mi trabajo profesional me atraen aquellas tareas y situaciones que implican un } \\
\text { desafío personal }\end{array}$ & 1 & 2 & 3 & 4 \\
\hline 11. No importa lo que me empeñe, a pesar de mis esfuerzos no suelo conseguir nada. & 1 & 2 & 3 & 4 \\
\hline 12. La mejor manera que tengo de alcanzar mis propias metas es implicándome a fondo. & 1 & 2 & 3 & 4 \\
\hline 13. A menudo trabajo duro, pues es la mejor manera para alcanzar mis propias metas & 1 & 2 & 3 & 4 \\
\hline $\begin{array}{l}\text { 14. La mayoría de las veces mis jefes o superiores toman en consideración lo que yo } \\
\text { tenga que decir }\end{array}$ & 1 & 2 & 3 & 4 \\
\hline 15. No me esfuerzo en mi trabajo, ya que, de cualquier forma, el resultado es el mismo. & 1 & 2 & 3 & 4 \\
\hline 16. Siento que si alguien trata de herirme, poco puedo hacer para intentar pararle. & 1 & 2 & 3 & 4 \\
\hline 17. En la medida que puedo trato de tener nuevas experiencias en mi trabajo cotidiano & 1 & 2 & 3 & 4 \\
\hline $\begin{array}{l}\text { 18. En mi trabajo me atraen preferentemente las innovaciones y novedades en los } \\
\text { procedimientos }\end{array}$ & 1 & 2 & 3 & 4 \\
\hline $\begin{array}{l}\text { 19. La mayoría de las veces no merece la pena que me esfuerce ya que haga lo que } \\
\text { haga las cosas nunca me salen }\end{array}$ & 1 & 2 & 3 & 4 \\
\hline 20. Aunque hagas un buen trabajo jamás alcanzarás las metas & 1 & 2 & 3 & 4 \\
\hline 21. Dentro de lo posible busco situaciones nuevas y diferentes en mi ambiente de trabajo & 1 & 2 & 3 & 4 \\
\hline
\end{tabular}


ESCALA DE ACONTECIMIENTOS VITALES ESTRESANTES.

Por favor, señale con un círculo sobre el número aquel o aquellos acontecimientos vitales que le han sucedido en el último año. Puede señalar todos los que considere necesarios.

1. Muerte del cónyuge

2. Divorcio

3. Separación matrimonial

4. Encarcelación

5. Muerte de un familiar cercano

6. Lesión o enfermedad personal

7. Matrimonio

8. Despido del trabajo

9. Paro

10. Reconciliación matrimonial

11. Jubilación

12. Cambio de salud de un miembro de la familia

13. Drogadicción y/o alcoholismo

14. Embarazo

15. Dificultades o problemas sexuales

16. Incorporación de un nuevo miembro a la familia

17. Reajuste de negocio

18. Cambio de situación económica

19. Muerte de un amigo íntimo

20. Cambio en el tipo de trabajo

21. Mala relación con el cónyuge

22. Juicio por crédito o hipoteca

23. Cambio de responsabilidad en el trabajo

24. Hijo o hija que deja el hogar

25. Problemas legales

26. Logro personal notable

27. La esposa comienza o deja de trabajar

28. Comienzo o fin de escolaridad

29. Cambio en las condiciones de vida

30. Revisión de hábitos personales

31. Problemas con el jefe

32. Cambio de turno o de condiciones laborales

33. Cambio de residencia

34. Cambio de colegio

35. Cambio de actividad de ocio

36. Cambio de actividad religiosa

37. Cambio de actividades sociales

38. Cambio de hábito de dormir

39. Cambio en el número de reuniones familiares

40. Cambio de hábitos alimentarios

41. Vacaciones

42. Navidades

43. Leves transgresiones de la ley

Recibido: 16 de octubre 2015;

Aceptado: 22 de enero 2016

ISSN 1695-6141

(C) COPYRIGHT Servicio de Publicaciones - Universidad de Murcia 